\title{
CATALYTIC COMBUSTION OF HYDROCARBONS OVER PEROVSKITES
}

\author{
Lucio Forni* and I.Rossetti \\ Dipartimento di Chimica Fisica ed Elettrochimica, Università di Milano \\ Via Golgi, 19 I-20133 Milano, Italy \\ Fax :+39-02-70638129; E-mail: I.forni@csrsrc.mi.cnr.it
}

\begin{abstract}
The advantages and disadvantages of the catalysts so far employed or proposed for the low-temperature catalytic combustion of hydrocarbons, in both static and mobile energy production devices, are discussed. Furthermore, $\mathrm{L} \mathrm{La}_{0.9} \mathrm{Ce}_{0.1} \mathrm{CoO}_{3 \pm \delta}$ perovskite has been prepared by a recently proposed new flame-hydrolysis method. This proved a high surface area, thermally highly resistant catalyst. The partial substitution of $\mathrm{Ce}$ for $\mathrm{La}$ in such a cobaltite led to a relatively low suprafacial activity, but to a high bulk oxygen mobility, leading to high intrafacial activity for the catalytic flameless combustion of methane. The best operating conditions have been also found, for supporting the so prepared active phase, by dip-coating of a cordieritic honeycomb support, after deposition of an alumina primer. A very active and durable catalyst was so obtained, useful for practical application in the environmentally friendly low-temperature combustion of methane.
\end{abstract}

Keywords:

Perovskite catalyst; Flame-hydrolysis technique; Methane catalytic flameless combustion; Honeycomb support; Dip-coating.

\section{INTRODUCTION}

The abatement of harmful pollutants such as $\mathrm{CO}, \mathrm{NO}_{x}$ and unburned hydrocarbons in combustion exhausts of any source is of ever growing concern. In particular, it is wellknown that, due to thermodynamic reasons, the catalytic flameless combustion (CFC) of hydrocarbons $(\mathrm{HC})$ can virtually suppress the formation of these species, being carried out at substantially lower temperature (below $800^{\circ} \mathrm{C}$ ) with respect to usual combustion. So far, the catalysts suggested and employed for CFC reactions are based on supported noble metals. However, besides being expensive, the latter easily sinterise and can form even more harmful volatile compounds. Among the possible substitutes for noble metals, perovskite-type transition metal oxide mixtures, when properly prepared, showed comparably active, highly resistant to deactivation and much cheaper.

Perovskites are mixed oxides of general formula $A B O_{3 \pm \delta}$, where $A$ is usually a lanthanide ion and $B$ is a transition metal ion. Both $A$ and $B$ can be partially substituted, leading to a wide variety of mixed oxides of general formula $A_{1-x} A^{\prime}{ }_{x} B_{1-y} B_{y}^{\prime} O_{3 \pm \delta}$, characterised by structural and electronic defects, owing to their non-stoichiometry, indicated by the $\delta$ subscript in the formula. For full oxidation reactions, such as CFC, the cation $B$ is considered the responsible of catalytic activity [1], while the cation A, especially when partially substituted with a cation $A^{\prime}$ of different valence, governs the formation of crystal lattice vacancies and can stabilise unusual oxidation states for $B$, leading to different catalytic performance [1-3]. The oxidation activity of perovskites has been ascribed to their ionic conductivity, to oxygen mobility within their lattice [2], to their reducibility and to their oxygen sorption properties [4]. Indeed, two possible mechanisms, 
a suprafacial and an intrafacial one, were proposed since long time [5] and now widely accepted for oxidation reactions over these catalysts. The former mechanism rises from the interaction of surface oxygen with reactants and it is operative at low temperature $\left(<400^{\circ} \mathrm{C}\right)$. The latter is effective at higher temperature $\left(>400^{\circ} \mathrm{C}\right)$ and involves a Mars-Van Krevelen redox cycle. In it bulk oxygen migrates towards the surface, becoming available for the oxidation of the adsorbed substrate and it is quickly replaced by oxygen coming from the gaseous phase. The mobility of $\mathrm{O}^{2-}$ ions in the crystalline framework determines the mechanism of the catalytic reaction [3].

The preparation procedure is of fundamental importance to determine both activity and durability of the catalyst. Various preparation methods can be found in literature [410], but high surface area and thermal resistance to sintering are not easy to be obtained simultaneously by those procedures. For instance, the traditional calcination-milling (CM) route for preparing such materials, involving several CM cycles of the precursor powder mixture, ends in a low surface area, coarse-size particles of relatively low catalytic activity. Therefore, several techniques were developed, in order to overcome this problem, the most commonly used being the so-called sol-gel-citrate (SGC) method [8]. This leads to catalysts of high surface area (up to $50 \mathrm{~m}^{2} / \mathrm{g}$ ), so highly active, which, however, rapidly sinterise under the usual CFC conditions, due to the too low temperature $\left(<800^{\circ} \mathrm{C}\right)$ attained during preparation. A solution of the problem can be the synthesis of highly dispersed nanometre-size powders, obtainable by the flame hydrolysis (FH) technique. The latter was successfully applied to other materials, particularly to silica [11], which is currently prepared in very fine particles (e.g. Cab-o-Sil) from $\mathrm{SiCl}_{4}$. However, to our knowledge, the $\mathrm{FH}$ technique has been applied so far to oxides or to oxide mixtures, whose metals possess at least one volatile precursor $[12,13]$.

Recently $[3,10]$ we presented a new method for the preparation of perovskitic mixed oxides, based on $\mathrm{FH}$ of an aqueous solution of the precursor salts. The method succeeded in preparing nanometre-size particles of high surface area, by extending the $\mathrm{FH}$ technique to a multicomponent aqueous solution of the precursors, hydrolysed in a high-temperature flame. A series of high surface area catalysts were obtained [3,14], very active for the CFC of methane. In the present work the study was further extended on $\mathrm{La}_{0.9} \mathrm{Ce}_{0.1} \mathrm{CoO}_{3 \pm \delta}$, one of the most active catalyst for the CFC of hydrocarbons. The aim was to prepare this active phase by $\mathrm{FH}$ and to support it on commercial cordierite honeycomb, so to obtain a catalyst particularly suited for application in the environmentally friendly CFC of methane.

\section{EXPERIMENTAL}

\section{Catalyst Preparation}

A detailed description of our new $\mathrm{FH}$ procedure for catalyst preparation can be found elsewhere [10]. Briefly, the precursor salts (acetates or nitrates), in the desired ratios, were dissolved in $10 \% \mathrm{HNO}_{3}$. Citric acid was then added as a complexing agent, in $0.5 / 1$ molar ratio with respect to the sum of metal salts. The resulting solution was nebulised into a $\mathrm{H}_{2}+\mathrm{O}_{2}$ flame. The perovskite nanometric particles so formed were collected by means of a $10 \mathrm{kV}$ electrostatic precipitator. The estimated temperature of perovskite formation was $1600-1800^{\circ} \mathrm{C}$ and the residence time of the reagents in the hottest zone of the flame was a very few milliseconds. For the preliminary experiments of honeycomb-supporting, a sample of the same composition was prepared also by the SGC technique. 


\section{Catalyst characterisation}

BET surface area and porosity were determined by means of a Micromeritics ASAP 2010 instrument. XRD analysis was done on a Philips PW1820 powder diffractometer, by using the Ni-filtered $\mathrm{Cu} \mathrm{K \alpha}$ radiation $(\lambda=0.15418 \mathrm{~nm})$. Solid phases were identified by comparison of XRD patterns with literature data [15]. Morphology and particle size were determined by means of a Cambridge Stereoscan 150 Scanning Electron Microscope (SEM). The composition of every sample was determined by X-ray Fluorescence (XRF), on a Jordan-Valley EX310 instrument, calibrated by analysing oxide mixtures of known composition.

\section{Catalytic activity tests}

The bench-scale continuous apparatus for testing activity for the CFC of methane was centred on a continuous, downflow quartz reactor. $0.2 \mathrm{~g}$ of catalyst, pressed into wafers, then ground and sieved to $0.15-0.25 \mathrm{~mm}$ particles, were loaded, after dilution with $1.3 \mathrm{~g}$ of quartz of the same particle size. The void space above and below the catalyst bed was filled with quartz beads $(0.25-0.85 \mathrm{~mm})$. The catalyst was activated by flowing 20 $\mathrm{cm}^{3} / \mathrm{min}$ of air, while increasing temperature $\left(10^{\circ} \mathrm{C} / \mathrm{min}\right)$ up to $600^{\circ} \mathrm{C}$, then kept for $1 \mathrm{~h}$. After cooling in flowing air down to $250^{\circ} \mathrm{C}$, the activity tests were carried out by feeding 10 $\mathrm{cm}^{3} / \mathrm{min}$ of a mixture of $1.04 \mathrm{vol} . \% \mathrm{CH}_{4}$ in $\mathrm{He}$, together with $10 \mathrm{~cm}^{3} / \mathrm{min}$ of air, while increasing temperature $\left(2^{\circ} \mathrm{C} / \mathrm{min}\right)$ from $250^{\circ} \mathrm{C}$ up to $600^{\circ} \mathrm{C}$. The outlet gas was analysed by means of an in-line HWD gas chromatograph (HP 5890).

\section{TPD-TPR-MS analysis}

A detailed description of the TPD-TPR-MS apparatus was given in a previous paper [16]. Briefly, the catalyst $(0.8 \mathrm{~g}, 0.15-0.25 \mathrm{~mm}$ particle size) was loaded into a continuous quartz microreactor, heated by an electric furnace controlled by an Eurotherm 822 TRC. Prior to every TPD or TPR run the catalyst was heated $\left(10^{\circ} \mathrm{C} / \mathrm{min}\right)$ up to $800^{\circ} \mathrm{C}$ in flowing He (purity $\geq 99.9999$ vol.\%).

TPD of pre-adsorbed oxygen was carried out in $\mathrm{He}$ as carrier gas, after saturation of the sample in flowing air at $750^{\circ} \mathrm{C}$, followed by cooling down to $50^{\circ} \mathrm{C}$. After switching the gas flow to $\mathrm{He}$, the catalyst was kept at $50^{\circ} \mathrm{C}$ for 10 minutes, then the temperature was raised $\left(10^{\circ} \mathrm{C} / \mathrm{min}\right)$ up to $800^{\circ} \mathrm{C}$, kept for 10 minutes. The composition of the outlet gas was monitored by means of a MKS PPT Residual Gas Analyser quadrupolar mass spectrometer (QMS).

A further TPD experiment was carried out in order to check the possible activation of the substrate. The catalyst was kept for $4 \mathrm{~h}$ at $250^{\circ} \mathrm{C}$ in a stream of $\mathrm{CH}_{4}(1.04 \mathrm{vol} . \%$ in $\mathrm{He}$ ) and then cooled down to $50^{\circ} \mathrm{C}$. The TPD run was then carried out in flowing $\mathrm{He}$ as described.

Three different TPR-MS experiments were performed, through either continuous or pulsed method. The first experiment was done by flowing the previously mentioned reaction gas mixture through the reactor, after saturating the sample in air as described. The second experiment was carried out by injecting into the He carrier gas, just before the reactor, $1.2 \mathrm{~cm}^{3}$ pulses of the same gas mixture, while running the same previously reported temperature programme. The third experiment was made by feeding continuously the same gas mixture, after pre-saturation of the sample in air. The MS species monitored were $\mathrm{CH}_{4}\left(\mathrm{~m} / \mathrm{z}=15\right.$, to avoid confusion with the $\mathrm{m} / \mathrm{z}=16$ fragment of $\mathrm{O}_{2}$ ), $\mathrm{H}_{2} \mathrm{O}(\mathrm{m} / \mathrm{z}=18), \mathrm{N}_{2}(\mathrm{~m} / \mathrm{z}=14$, to distinguish it from $\mathrm{CO}), \mathrm{O}_{2}(\mathrm{~m} / \mathrm{z}=32), \mathrm{CO}_{2}(\mathrm{~m} / \mathrm{z}=44)$, and $\mathrm{CO}$ $(\mathrm{m} / \mathrm{z}=28)$. 


\section{a) Preparation}

The perovskite catalyst was supported on the honeycomb by dip-coating, by means of a simple home-made apparatus [17], allowing to dip and to extract the support, at the desired rate, from the aqueous suspension of the catalyst powder. Indeed, the amount of material deposited onto the support depends on the extracting rate, at least within given limits: the higher the rate, the larger is the amount of suspension adhering to the honeycomb. The support, of cylindrical shape, $1 \mathrm{~cm}$ in diameter, $5 \mathrm{~cm}$ long, cut from a commercial $400 \mathrm{cpi}$ cordierite honeycomb, was precalcined at $500^{\circ} \mathrm{C}$ for $1.5 \mathrm{~h}$ and weighed. Then it was suspended to a thin nylon wire $(0.2 \mathrm{~mm}$ in diameter $)$, dipped into the suspension ( $4 \mathrm{wt} \%$ perovskite) and then extracted at a rate of $11 \mathrm{~cm} / \mathrm{min}$. The suspension was prepared by milling overnight at room temperature the perovskitic powder in 4 wt \% aq. nitric acid into a polypropylene jar with zirconia balls. A very homogeneous suspension was obtained, stable for several weeks. A preliminary dip-coating of the honeycomb with an alumina primer was needed, in order to ensure a better grafting of the catalytic material to the support. The suspension of primer was prepared similarly, by milling a mixture of $10 \mathrm{wt} \%$ boehmite (Disperal P2 ${ }^{\mathrm{TM}}$, kindly supplied by Condea Chemie) in $4 \mathrm{wt} \%$ aq. nitric acid. After coating either with the primer or with the perovskite, the honeycomb was left to dry at room temperature for $1 \mathrm{~h}$, calcined at $500^{\circ} \mathrm{C}$ for $1.5 \mathrm{~h}$ and weighed to determine the amount of coating material.

\section{b) Catalytic activity testing}

The previously described apparatus was employed, by simply substituting the quartz reactor with an Incoloy 800 reactor, the inner diameter of wich just fitted the external diameter of the honeycomb. The void space above and below the honeycomb was filled with the same $0.25-0.85 \mathrm{~mm}$ quartz beads, separated from the honeycomb by small flocks of quartz wool. The same catalyst activation and testing procedure was followed (vide supra).

\section{RESULTS AND DISCUSSION}

Composition and particle size of the perovskite samples obtained by the $\mathrm{FH}$ method can be controlled by varying four main parameters, while keeping constant the flow rate of $\mathrm{H}_{2}$ and $\mathrm{O}_{2}$ to the burner and the size of the latter and of the nebuliser nozzle [10]. The four parameters are: a) nature of the precursor salts, b) concentration of the latter in the aqueous solution, $c$ ) flow rate and $d$ ) humidity degree of the air fed to the nebuliser.

The nature of the precursor salts may hinder the formation of a clear solution of the desired composition and helps in keeping the $\mathrm{FH}$ to a sufficiently high temperature. This is the reason why organic anions, such as acetates, citrates, etc. have been preferred to inorganic ones, such as nitrates, chlorides, etc. The concentration of the solution governs the size of the perovskitic particles obtained. The higher the flow rate of the air fed to the nebuliser, the higher is the production rate of the process, but the lower becomes the flame temperature and hence the degree of crystallinity of the perovskite. Finally, a proper degree of humidity of the air sent to the nebuliser allows to keep constant the concentration of precursors solution during nebulisation, so keeping constant the particle size of the product and the production rate of the process. 


\section{Catalyst characterisation}

XRD analysis (Fig.1) of the FH-prepared sample confirmed the presence of a perfectly pure and crystalline perovskitic phase [15]. Its BET surface area was $18.6 \mathrm{~m}^{2} / \mathrm{g}$. This value falls between those usually obtained through the traditional SGC and CM preparation procedures, but it can be considered quite high, when taking into account the very high calcination temperature (over $1600^{\circ} \mathrm{C}$ ) attained during the $\mathrm{FH}$. The compromise between high surface area and high calcination temperature is made possible by the formation of nano-particles. Indeed, SEM analysis (Fig.2) showed that the present catalyst consisted of 20-60 nm spheroidal particles, usually agglomerated into 100-500 nm clusters. By decreasing the concentration of the precursors' solution and/or the diameter of the nebulisers' nozzle, a further decrease of particle size can be obtained [10], so increasing the catalyst surface area. However, this would be accompanied by a strong decrease of process productivity. Therefore, a compromise had to be reached also among these experimental parameters.

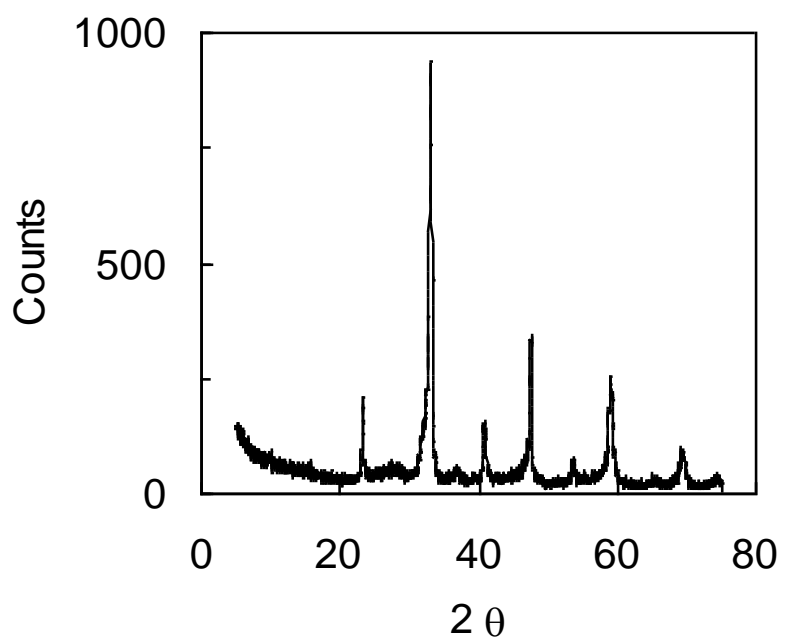

Fig.1 - XRD pattern of the FHprepared $\mathrm{La}_{0.9} \mathrm{Ce}_{0.1} \mathrm{CoO}_{3 \pm \delta}$ perovskite.

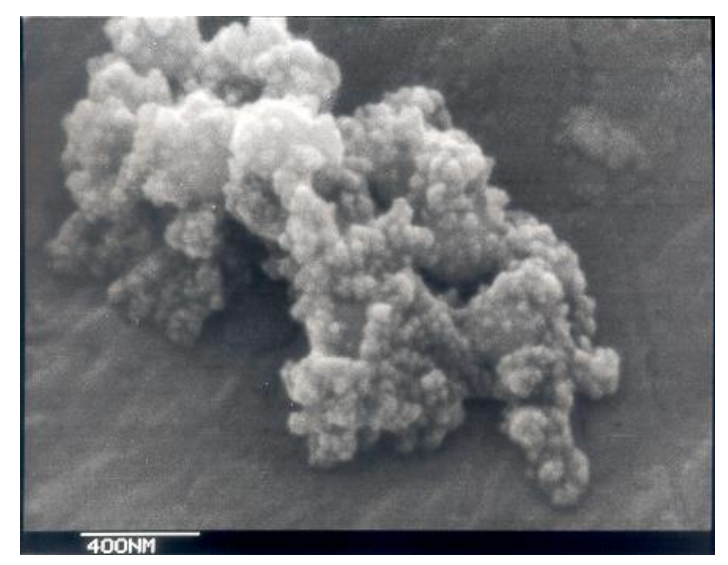

Fig.2 - Typical SEM micrograph of the $\mathrm{FH}$-prepared $\mathrm{La}_{0.9} \mathrm{Ce}_{0.1} \mathrm{CoO}_{3 \pm \delta}$ perovskite.
Qualitative XRF analysis excluded the presence of any extraneous element. Quantitative analysis confirmed, within the experimental error $( \pm 1 \mathrm{wt} \%)$, a good correspondence between actual composition and the nominal one, calculated from the weighed amounts of precursor salts.

\section{Catalytic activity}

The results of the activity test on the catalyst in powder form are shown in Fig.3. The values of $T_{0}, T_{50}$ and $T_{100}$, representing the temperature of light-off, of half-transformation and of complete conversion of methane, were $<250,438$ and $560^{\circ} \mathrm{C}$, respectively. The catalyst showed a relatively low activity at low temperature $\left(<400^{\circ} \mathrm{C}\right)$, to be attributed to a relatively high ionic mobility within the lattice. Indeed, it is well known $[1,3]$ that when oxygen mobility is low, the suprafacial mechanism is usually active, while a high ionic mobility favours the participation of bulk oxygen, so that the intrafacial mechanism (active at $\mathrm{T}>400^{\circ} \mathrm{C}$ ) is preferred.

A life test was also carried out, by comparing the performance of the present catalyst with that of a sample of the same composition, but prepared by the SGC method. After attaining the temperature $\mathrm{T}_{100}$ of methane complete conversion, the reaction was continued by keeping temperature at the $T_{100}$ value for at least 100 hours on-stream. The FH-prepared catalyst 
(Fig.4) proved much more resistant, by keeping 100\% methane conversion for the whole duration of the text, while the SGC-prepared sample started to decay rather rapidly after less than 40 hours on-stream. $S_{\text {BET }}$ data, collected on both the samples before and after this test, confirmed that the activity decay was due to the progressive sintering of the catalyst. Indeed, the surface area of the SGC-prepared sample decreased at a rate comparable with that of performance decay, while the $\mathrm{S}_{\mathrm{BET}}$ of the $\mathrm{FH}$-prepared catalyst remained unchanged.

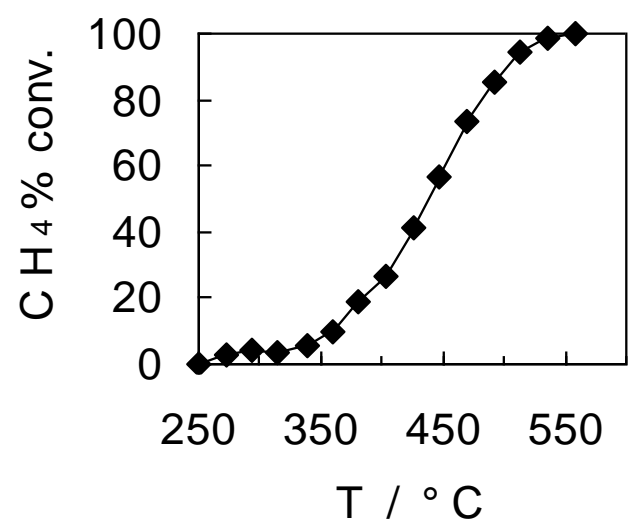

Fig.3 - Mol \% conversion of methane vs. reaction temperature under standard reaction conditions (see text). $\mathrm{FH}$-prepared $\mathrm{La}_{0.9} \mathrm{Ce}_{0.1} \mathrm{CoO}_{3 \pm \delta}$ perovskite in powder form.

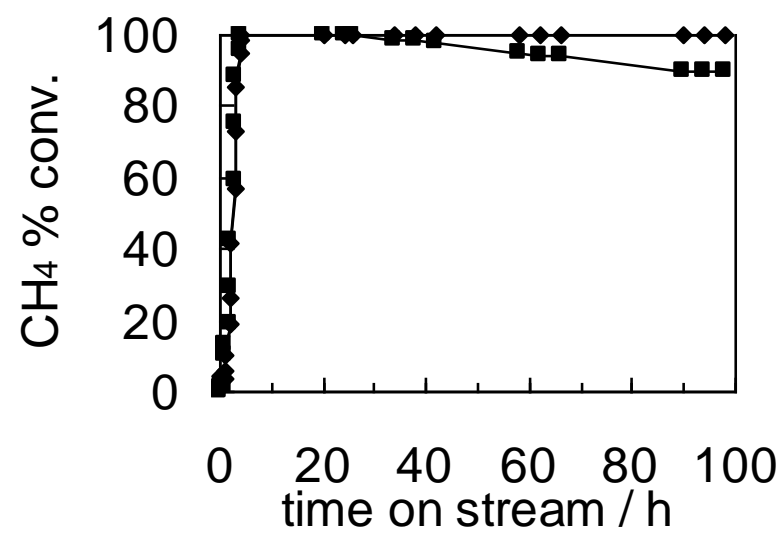
method.

Fig.4 - Life test for the catalyst prepared by the FH ( $\bullet)$ and by the SGC ( $\bullet)$

\section{TPD-MS analysis}

It is well-known [9] that, when a perovskite is heated in inert atmosphere at high temperature, oxygen vacancies can form. By cooling in air, these vacancies are filled with adsorbed oxygen and the charge compensation is provided by partial oxidation of the metal B ion. A TPD analysis after this pre-treatment reveals two possible desorption peaks, called $\alpha$ and $\beta$, widely reported in literature $[1,3,7-9,18]$. The $\alpha$ peak, attributed to the desorption of oxygen adsorbed on the catalyst surface, is not always observable, depending on the concentration of filled oxygen surface vacancies. In particular, its onset temperature and intensity depend partially on the nature of metal $B$, but mainly on the substitution degree of the $A$ ion with a cation of different valence $[3,7]$. The $\beta$ peak, characterised by higher onset temperature, depends tightly on the nature of the B ion, being correlated to its partial reduction to a lower oxidation state $[7,9]$. The onset 
temperature of the $\beta$ peak is usually very near to that of thermal decomposition of the pure B metal oxide.

The catalyst here prepared showed a TPD pattern (Fig.5) with a modest $\alpha$ peak around $330^{\circ} \mathrm{C}$, while the onset temperature of the $\beta$ peak was approximately $650^{\circ} \mathrm{C}$. Furthermore, the maximum of the $\beta$ desorption peak was not reached within the upper temperature limit $\left(800^{\circ} \mathrm{C}\right)$ of our apparatus. An intense $\alpha$ peak indicates a high concentration of oxygen surface vacancies. Hence, one may conclude that the present catalyst possesses a relatively low concentration of such vacancies. On the other hand, the presence of the $\beta$ peak and the value of its onset temperature can be considered a qualitative index of catalyst reducibility and of oxygen mobility within the bulk. Both these parameters are fundamental to describe catalytic activity. Indeed, according to the intrafacial mechanism [5], a redox cycle involving metal $B$ is responsible both of the substrate oxidation and of the reoxidation of the metal, back to the original valence, by the gaseous $\mathrm{O}_{2}$ present in the reaction atmosphere. Therefore, a low-temperature $\beta$ peak indicates an easy reducibility of the $B$ metal and hence an enhanced redox property of the catalyst and a quick oxygen transport through the perovskitic lattice. Oxygen mobility is tightly connected with the concentration of oxygen bulk vacancies: the higher their concentration, the lower is the activation energy for oxygen transport. A very low oxygen mobility was observed with the La-unsubstituted $\mathrm{LaCoO}_{3 \pm \delta}$ perovskite [14], while an ionic conductivity increase is here noticed after $10 \%$ Ce substitution for La. It may be concluded that the present catalyst, characterised by the presence of a modest $\alpha$ peak, is predominantly active through the intrafacial mechanism.

In addition to these $\mathrm{O}_{2}$ TPD experiments, we made also a TPD analysis after preadsorption of methane. No $\mathrm{m} / \mathrm{z}=15$ desorption signal was observed, indicating that methane did not adsorb in appreciable amount and, hence, that catalytic activity was connected with oxygen mobility, rather than with the direct activation of the substrate to be oxidised.

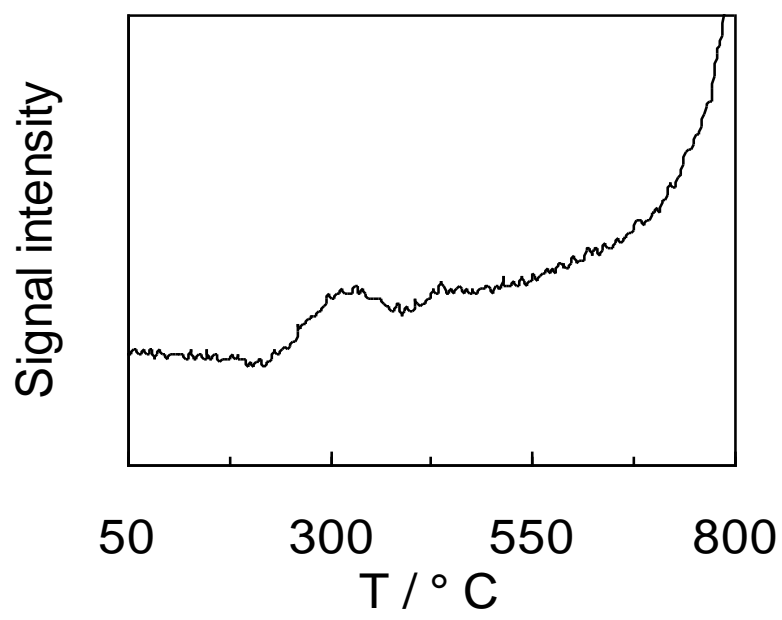

Fig.5 - TPD-MS spectra of oxygen $(\mathrm{m} / \mathrm{z}=32)$ preadsorbed onto the FH-prepared $\mathrm{La}_{0.9} \mathrm{Ce}_{0.1} \mathrm{CoO}_{3 \pm \delta}$ perovskite.

\section{TPR-MS analysis}

The results of the pulsed and of continuous TPR-MS experiments, carried out by feeding the mixture of $\mathrm{CH}_{4}(1.04 \%$ in $\mathrm{He})+$ air, matched almost perfectly. Both the suprafacial and the intrafacial reaction mechanisms have been observed also through these experiments, though less clearly. The TPR-MS experiments, carried out by feeding only $\mathrm{CH}_{4}$ after $\mathrm{O}_{2}$ pre-adsorption, allowed us to determine the possible influence of the 
different ( $\alpha$ and $\beta$ ) oxygen species on catalytic activity. The catalyst began to be active at ca. $350^{\circ} \mathrm{C}$ (Fig.6) and conversion attained almost $100 \%$ at ca. $750^{\circ} \mathrm{C}$. Hence, bulk oxygen only seems to be involved in the reaction, the species adsorbed on the surface seeming practically uninvolved. Very likely, $\alpha$ desorption takes place at a too low temperature to be useful for the reaction. Indeed, when the TPR experiment was carried out by feeding $\mathrm{CH}_{4}$ + air, the surface sites set free after $\alpha$ desorption could be filled by new oxygen species coming from the gaseous phase and readily activated. So, when the onset temperature for the reaction was reached, activated oxygen was available. By contrast, in the absence of oxygen in the reaction atmosphere, the light-off temperature was shifted towards higher values, i.e. towards the onset temperature of oxygen $\beta$ desorption.

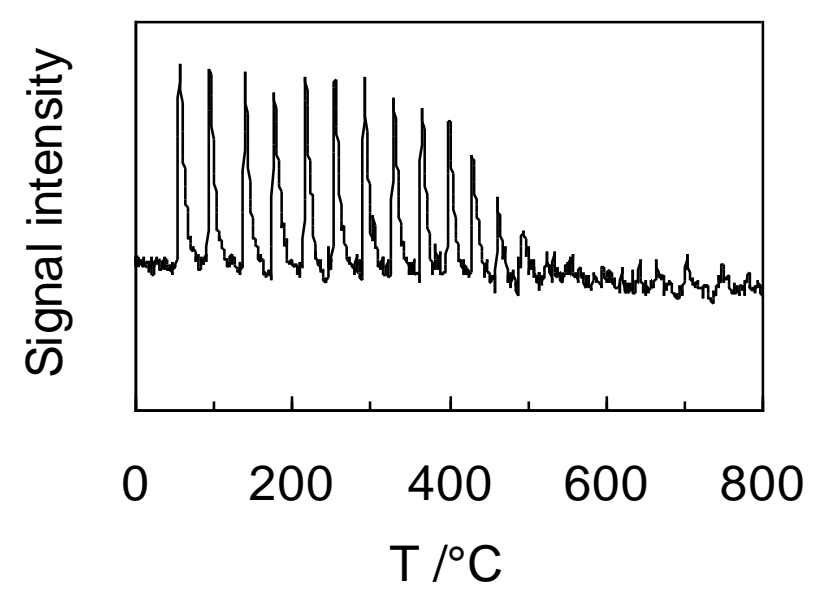

Fig.6 - Activity for methane oxidation of the $\mathrm{FH}$-prepared $\mathrm{La}_{0.9} \mathrm{Ce}_{0.1} \mathrm{CoO}_{3 \pm \delta}$ perovskite, as monitored by TPR-MS $(\mathrm{m} / \mathrm{z}=15)$.

\section{Honeycomb-supported catalys}

Some preliminary experiments, by employing the SGC-prepared perovskite, aimed at tuning the coating procedure. These experiments allowed to define the most favourable conditions for the preparation of the coating suspension of both primer and perovskite and for the dip-coating procedure, namely concentration of the suspension and of the peptizing agent, dipping and extracting rate, drying and calcination temperature. In addition, they showed that in the absence of primer the activity per unit weight of perovskite was by ca. 30\% lower for the honeycomb-supported than for the unsupported catalyst. By contrast, in the presence of primer, the unsupported and supported samples performed similarly. Furthermore, in the absence of primer and/or for excessive loading of the active phase, the grafting of the latter to the support showed unacceptably weak.

Table 1. - Composition of the honeycomb-supported catalysts.

\begin{tabular}{llll}
\hline $\begin{array}{l}\text { Sample } \\
\text { No. }\end{array}$ & $\begin{array}{l}\text { Uncoated } \\
\text { honeycomb } \\
\text { weight / g }\end{array}$ & $\begin{array}{l}\text { Primer } \\
\text { loading } \\
\text { wt \% }\end{array}$ & $\begin{array}{l}\text { Perovskite } \\
\text { loading } \\
\text { wt \% }\end{array}$ \\
\hline 1 & 1.53 & 5.8 & 1.6 \\
2 & 1.54 & 4.6 & 3.8 \\
3 & 1.56 & 5.1 & 5.6
\end{tabular}




\begin{tabular}{llll}
4 & 1.59 & 2.4 & 3.1 \\
5 & 1.54 & 4.6 & 3.6 \\
6 & 1.63 & 7.4 & 2.8 \\
\hline
\end{tabular}

Two series of catalysts were then prepared with the $\mathrm{FH}$-made perovskite, with different loading of either primer or perovskite, respectively, aiming at defining the best loading of both these components. The composition of these samples is shown in Table 1. Their activity was compared under identical experimental conditions. After activation of the catalyst in air (vide supra) the activity tests were carried out by feeding a gas mixture composed of $50 \%$ (vol.) of air and $50 \%$ of 1.04 vol.\% $\mathrm{CH}_{4}$ in $\mathrm{He}$, while increasing temperature $\left(2^{\circ} \mathrm{C} / \mathrm{min}\right)$ from $250^{\circ} \mathrm{C}$ up to $600^{\circ} \mathrm{C}$. Time factor $(\tau=$ perovskite mass $/$ gas flow rate) was kept at the same value for all of the runs, by adapting the overall gas flow rate to the amount of perovskite present in the honeycomb under test. This has been done in order to stress the effect of the loading of primer and of active phase on the specific activity of the perovskite, i.e. on the activity referred to unit weight of active phase.

Some preliminary tests confirmed the effect of primer reported for the SGCprepared, honeycomb-supported perovskite (vide supra). Indeed, in the absence of primer activity of supported perovskite showed lower than that of the unsupported sample, while in the presence of primer supported and unsupported perovskites performed comparably.

The activity data for the two series of Table 1 samples are collected in Fig.7 and 8. $T_{0}, T_{50}$ and $T_{100}$ appear affected by loading, though to a different extent. Indeed, the effect of primer (Fig.7) seems to level off or even to worsen activity for $\geq 5 \mathrm{wt} \%$ loading. This means that such a loading should correspond to some saturation of the support surface by the alumina primer. By contrast, $T_{0}, T_{50}$ and $T_{100}$ steadily decrease with increasing the loading of perovskite (Fig.8), showing that activity increases with increasing loading. This means that the here investigated upper limit of loading lies below the saturation of the support surface by the active phase. However, since higher loading of the latter led to lower grafting strength of the perovskitic coating, a value around $6 \mathrm{wt} \%$ should correspond to a good compromise between these two contrasting needs.

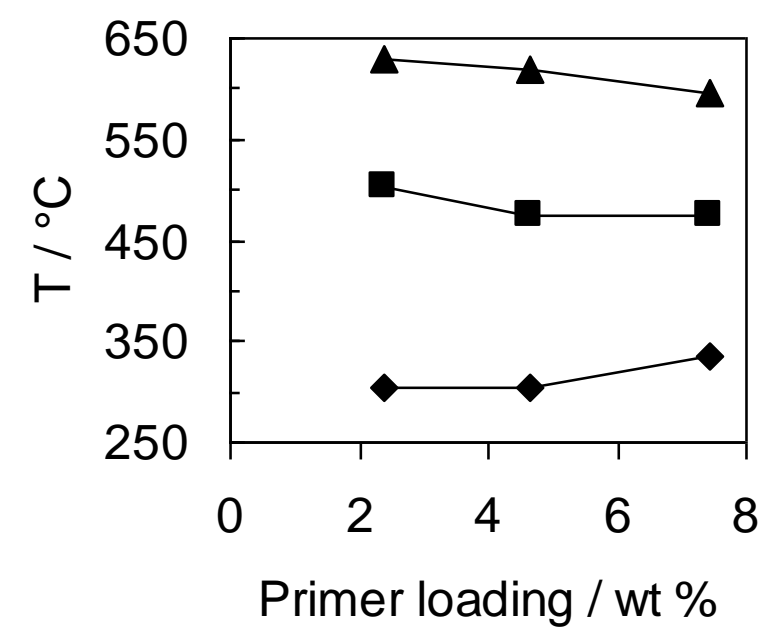

Fig.7 - Trend of $(\bullet) \mathrm{T}_{0},(\boldsymbol{\square}) \mathrm{T}_{50}$ and $(\boldsymbol{\Delta}) \mathrm{T}_{100}$ for methane CFC vs. loading of primer on the honeycomb at constant perovskite loading (catalysts 4 to 6 , Table 1). 
At last, a life test on sample No.3 (Table 1), carried out for over 110 hours onstream at its $T_{100}$ value of $575^{\circ} \mathrm{C}$, confirmed the very good activity, stability and resistance of the present best catalyst, at least within the time limit of our durability run.

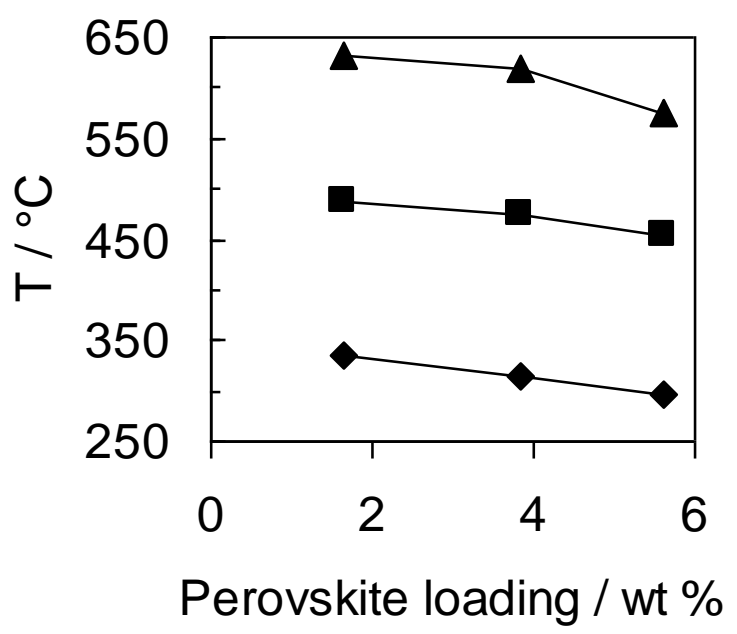

Fig.8 - Trend of $(\bullet) \mathrm{T}_{0},(\boldsymbol{\nabla}) \mathrm{T}_{50}$ and $(\boldsymbol{\Delta}) \mathrm{T}_{100}$ for methane CFC vs. loading of perovskite on the honeycomb at constant primer loading (catalysts 1 to 3 , Table 1).

\section{CONCLUSIONS}

Our $\mathrm{FH}$ preparation method led to a La-Ce-Co-based perovskitic active phase characterised by high crystallinity, high surface area and high activity for the CFC of methane, as well as by high thermal resistance.

In this cobaltite the partial substitution of $\mathrm{Ce}$ for La led to a low suprafacial, lowertemperature activity, confirmed by the presence of a weak oxygen $\alpha$ desorption peak, and to a high oxygen mobility, leading to a high intrafacial, higher-temperature activity. Furthermore, no appreciable adsorption of methane was observed, indicating that catalytic activity is connected essentially with bulk oxygen mobility, rather than to direct activation of the hydrocarbon.

The best conditions have been defined for supporting the so prepared active phase on cordieritic honeycomb, by dip-coating after deposition of an alumina primer. A promising highly active and durable catalyst was so obtained, useful for practical application in the environmentally friendly catalytic flameless combustion of methane.

\section{ACKNOWLEDGEMENTS}

The financial aid of the Italian National Council for Research (CNR) through the PF-MSTA-II, Contract No. 99.01789.PF 34, is gratefully acknowledged. We are indebted to G.Gianotto for his valuable help in setting up the dip-coating apparatus. 


\section{REFERENCES}

[1] - N. Yamazoe, Y. Teraoka, Catal. Today, 8 (1990) 175.

[2] - M. S. Islam, M. Cherry, C. R. A. Catlow, J. Solid State Chem., 124, (1996), 230.

[3] - R. Leanza, I. Rossetti, L. Fabbrini, C. Oliva, L. Forni, Appl. Catal. B: Environmental, 28 (2000) 55.

[4] - L.G.Tejuca, J.L.G.Fierro, Eds., Properties and Applications of Perovskite-type Oxides, Dekker, New York, 1993.

[5] - R.J.H. Voorhoeve, J.P. Remeika and D.W. Johnson, Science, 180 (1973) 62.

[6] - J.Twu and P.K.Gallagher, in Properties and Applications of Perovskite-Type Oxides, Ed. L. G. Tejuca, J. L. G. Fierro, Dekker, New York, 1993, p. 1.

[7] - Y. Teraoka, H.M. Zhang, N. Yamazoe, Chem. Lett., 1985, 1367.

[8] - M.S.G.Baythoun and F.R.Sale, J. Mater. Sci., 17 (1982) 2757.

[9] - H.M. Zhang, Y. Shimizu, Y. Teraoka, N. Miura, N. Yamazoe, J. Catal., 121 (1990) 432.

[10] - R. A. M. Giacomuzzi, M. Portinari, I. Rossetti, L. Forni, Stud. Surf. Sci. And Catal., A. Corma, F.V. Melo, S. Mendioroz, J.L.G. Fierro, Eds., Vol. 130, Elsevier, Amsterdam, 2000, p.197.

[11] - J. Long and S. J. Teichner, Rev. Hautes Temper. et Refract., 2 (1965) 47.

[12] - W.R.Moser, J.D.Lennhoff, J.E.Cnossen, K.Fraska, J.W.Schoonover and J.R.Rozak, in Advanced Catalysts and Nanostructured Materials, W.R.Moser, Ed., Academic Press, New York, 1996, p.535.

[13] - W.J.Stark, K.Wegner, S.E.Pratsinis and A.Baiker, J.Catal., 197 (2001) 182.

[14] - I.Rossetti and L.Forni, Appl. Catal., B: Environmental, submitted.

[15] - Selected Powder Diffraction Data, Miner. DBM (1-40), J.C.P.D.S., Swarthmore, PA, 1974-1992.

[16] - L.Forni, M. Toscano and P. Pollesel, J. Catal., 130 (1991) 392.

[17] - G.Gianotto, Thesis, Industrial Chemistry degree, Università di Milano, March 2001.

[18] - D.Ferri and L.Forni, Appl. Catal., B: Environmental, 16 (1998) 119. 